\title{
An Embedding Theorem for Abelian Monoidal Categories
}

\author{
PHÙNG HỒ HẢI \\ Hanoi Institute of Mathematics, PO Box 631, 10000 Bo Ho, Hanoi, Vietnam. \\ e-mail:phung@thevinh.ncst.ac.vn
}

(Received: 6 June 2000; accepted in final form: 6 April 2001)

\begin{abstract}
We show that, with some technical conditions, an Abelian monoidal category admits a monoidal embedding into the category of bimodules over a ring. The case of semisimple rigid monoidal categories is studied in more detail.
\end{abstract}

Mathematics Subject Classifications (2000). Primary: 18D10, 18E20, Secondary 16D20, $16 \mathrm{~W} 30$

Key words. embedding theorem, monoidal category.

\section{Introduction}

The problem of finding an embedding theorem for Abelian monoidal categories is motivated on the one hand by the Freyd-Mitchell full embedding theorem and on the other hand by Deligne's and Doplicher and Roberts' theories of 'abstract Tannakian-Krein's duality' ([3, 4]).

By definition, an embedding from one Abelian category to another is a faithful functor which sends nonzero objects to nonzero objects. According to Freyd and Mitchell, a small Abelian category admits an exact full embedding into the category of modules over a ring [11]. This important theorem allows one, for example, to treat finite diagrams in an Abelian category as diagrams of modules.

In [3], Deligne shows that, under certain technical conditions, an Abelian symmetric rigid monoidal category admits an exact monoidal embedding into the category of modules over a commutative ring. Hence, by Tannaka-Krein duality, such a category is equivalent to the category of representations of a groupoid [3, Theorem 1.12]. Doplicher and Roberts study the case of compact groups and obtain an analogous result for $C^{*}$-categories [4].

With the birth of quantum groups ([5]), the theory of monoidal category has a new motivation. The 'symmetric' condition turns out to be too strong and is replaced by a weaker one, the 'braided' condition. The problem of generalizing Deligne's and Doplicher and Roberts' results to braided categories is interesting. Yet, one does not know what can be a target for such an embedding. 
The situation seems to be simpler if we drop the symmetry, i.e., to find an embedding for Abelian monoidal categories. A natural candidate for the target category is the category of bimodules over a ring. In this paper we show that any small monoidal cateory with an exact tensor product admits a right exact monoidal embedding into the category of bimodules over a ring. In particular, a small Abelian rigid monoidal category admits an exact monoidal embedding (Theorem 3.2).

Unfortunately, this embedding theorem does not seem to help solve the problem for braided categories, for, according to Schauenburg [13], the category of bimodules over a ring does not generally possess a braiding. Indeed, he showed that the center of the category of bimodules over a ring is trivial, i.e., contains only direct sums of the ring.

Let us briefly explain the main idea of the paper. To find an embedding for a small Abelian monoidal category, we first extend it to a larger monoidal category which is cocomplete and has an injective cogenerator, namely, a Grothendieck monoidal category. Then we extend the latter category to a module category, say over a ring $R$. Finally, we construct a monoidal functor from a module category with a monoidal structure to a bimodule category with the usual tensor product. The construction of the last functor will be given in Section 2. In Section 3 we explain how to extend the tensor product on a small Abelian monoidal category to a monoidal structure on a module category containing this small Abelian monoidal category. In the last section, we consider an application to the special case of small semisimple categories. An explicit embedding is described. As a consequence, we show that a small semisimple symmetric monoidal category with a simple unit object is Tannakian (Corollary 4.5).

Throughout the paper, the tensor product over a ring $R$ is denoted for short by $\otimes$. $\otimes$ also denotes the tensor product in an abstract monoidal category when no confusion may appear, othewise, we use the signs $\odot$ or $\square$. The category of right $R$-modules (resp. left $R$-modules or $R-R$-bimodules) is denoted by $\operatorname{Mod}_{R}$ (resp. ${ }_{R} \operatorname{Mod}$ or ${ }_{R} \operatorname{Mod}_{R}$ ). $\operatorname{Hom}_{R}$ (resp. ${ }_{R}$ Hom or ${ }_{R} \mathrm{Hom}_{R}$ ) denotes the set of homomorphisms of right $R$-modules (resp. left $R$-modules or $R-R$-bimodules).

\section{Abelian Monoidal Categories}

\subsection{MONOIDAL CATEGORIES}

Let $A$ be a category. A monoidal structure on $A$ consists of the following data: a bifunctor

$$
\otimes: \mathrm{A} \times \mathrm{A} \longrightarrow \mathrm{A},(X, Y) \longmapsto X \otimes Y
$$

called the tensor product, an object $I$, called the unit object, for which 
(i) there exists a natural isomorphism $\alpha$ between the functors

$$
(-\otimes-) \otimes-\quad \text { and } \quad-\otimes(-\otimes-), \alpha_{X, Y, Z:}(X \otimes Y) \otimes Z \longrightarrow X \otimes(Y \otimes Z),
$$

called the associativity constraint, such that the following diagram commutes:

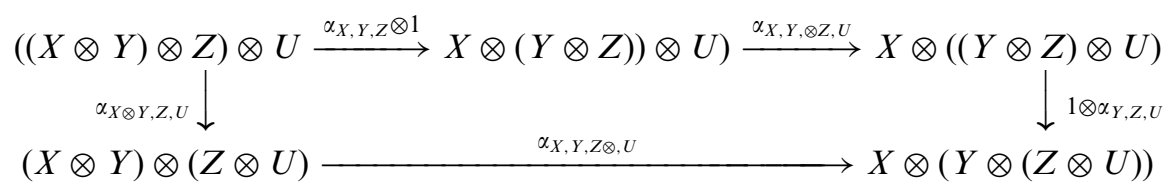

(2) there exist natural ismorphisms of functors $-\otimes I, I \otimes-$ with the identity functor: $\rho_{X}: X \otimes I \longrightarrow X$ and $\lambda_{X}: I \otimes X \longrightarrow X$, called right and left units, such that the following diagram commutes:

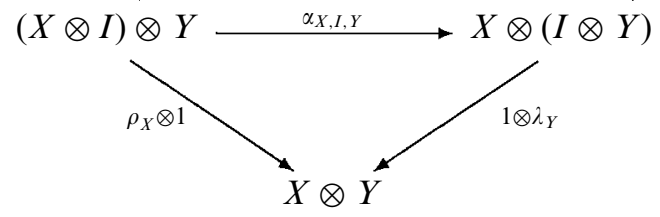

$(\mathrm{A}, \otimes, I, \alpha, \lambda, \rho)$ is called a monoidal category. In the case when $\alpha, \lambda, \rho$ are identity morphisms, we have a strict monoidal category. In the general case, the associativity constraint $\alpha$ allows one to speak of a tensor product of many objects $X_{1} \otimes X_{2} \otimes \cdots \otimes X_{n}$, without specifying the order in which the tensor product is applied (Mac Lane's coherence theorem [9, VII,2]).

Using (1) and (2), we can show that the following diagrams commute:

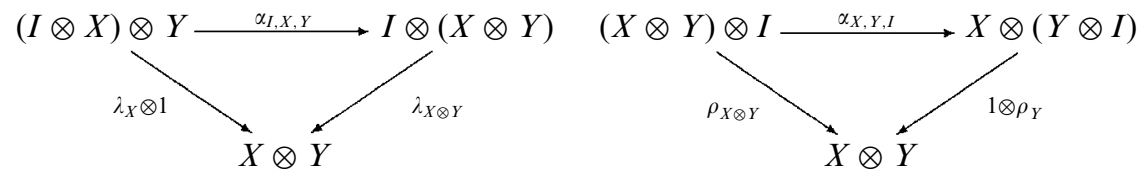

and that $\lambda_{I}=\rho_{I}$ (cf. [12]).

From the definition of a bifunctor, we have

$$
f \otimes g=(1 \otimes g) \circ(f \otimes 1)=(f \otimes 1) \circ(1 \otimes g) .
$$


In particular, using the isomorphism $\lambda_{I}=\rho_{I}$, we have, for $r, s \in \operatorname{End}(I)$ :

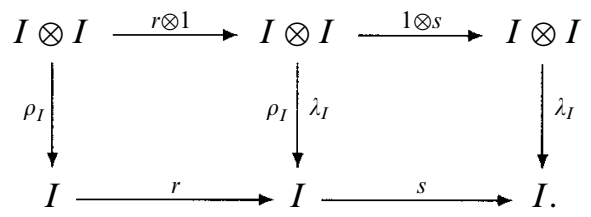

Therefore, using Equation (3), we have $r \circ s=s \circ r$. Thus, End( $I$ ) is an Abelian group with respect to composition. Further, this group acts on any set $\operatorname{Hom}(X, Y)$ from the left and the right by means of the isomorphism $\lambda$ and $\rho$ :

$$
r \cdot f:=\lambda_{Y}(r \otimes f) \lambda_{X}^{-1}, \quad f \cdot r:=\rho_{Y}(f \otimes r) \rho_{X}^{-1} .
$$

We have $1_{I} \cdot f=f \cdot 1_{I}=f$.

\subsection{THE INTERNAL HOMS}

Each object $X$ in A defines a functor $X \otimes-: \mathrm{A} \longrightarrow \mathrm{A}, Y \longmapsto X \otimes Y$. If this functor has a right adjoint, the right adjoint will be denoted by $\operatorname{rhom}(X,-)$. We have, by definition, a natural isomorphism

$$
\operatorname{Hom}(X \otimes Y, Z) \cong \operatorname{Hom}(Y, \operatorname{rhom}(X, Z)), \quad \forall Y, Z .
$$

The functor Ihom is define analogously by

$$
\operatorname{Hom}(Y \otimes X, Z) \cong \operatorname{Hom}(Y, \operatorname{lnom}(X, Z)), \quad \forall Y, Z \text {. }
$$

The category $A$ is called left closed (resp. right closed or closed) if the functor $\operatorname{lnom}(X,-)$ (resp. $\operatorname{rhom}(X,-)$ or both functors) is defined for any $X \in \mathrm{A}$. The general theory of adjoint functors (cf. [11, Corollary V.3.2]) gives us the following criteria of closedness

LEMMA 1.1. Assume that $\mathrm{A}$ is a cocomplete category with a generator. Then the tensor product on $\mathrm{A}$ is closed if and only if it commutes with colimits.

In the general case, the functor $\operatorname{Ihom}(X,-)$ and $\operatorname{rhom}(X,-)$ preserves colimits. If the category is closed, then we can also speak of the (contravariant) functors $\operatorname{lhom}(-, Z)$ and $\operatorname{rhom}(-, Z)$.

LEMMA 1.2. Assume that $\mathrm{A}$ is closed. Then the functors $\operatorname{Ihom}(-, Z)$ and $\operatorname{rhom}(-, Z)$ preserve colimits (i.e. sending colimits to limits). 
Proof. We have, for any $Y \in \mathrm{A}$,

$$
\begin{aligned}
\operatorname{Hom}\left(Y, \operatorname{rhom}\left(\lim _{\longrightarrow} X_{i}, Z\right)\right) & \cong \operatorname{Hom}\left(\lim _{\longrightarrow} X_{i} \otimes Y, Z\right) \\
& \cong \operatorname{Hom}\left(\underset{\lim }{\longrightarrow}\left(X_{i} \otimes Y\right), Z\right) \\
& \cong \lim _{\leftarrow} \operatorname{Hom}\left(X_{i} \otimes Y, Z\right) \\
& \cong \underset{\leftarrow}{\lim } \operatorname{Hom}\left(Y, \operatorname{rhom}\left(X_{i}, Z\right)\right) \\
& \cong \operatorname{Hom}\left(Y, \lim \operatorname{rhom}\left(X_{i}, Z\right)\right) .
\end{aligned}
$$

Since the above isomorphisms hold for any $Y$, we conclude that

$$
\operatorname{rhom}\left(\lim _{\longrightarrow} X_{i}, Z\right) \cong \lim _{\leftarrow} \operatorname{rhom}\left(X_{i}, Z\right)
$$

The case of $\operatorname{lnom}(-, Z)$ is treated quite analogously.

\subsection{RIGID OBJECTS}

Setting $Z=I$ and $Y=\operatorname{lnom}(X, I)$ in (8), we obtain a morphism ev : $_{X} \operatorname{Ihom}(X, I) \otimes$ $X \longrightarrow I$, which corresponds to the identity morphism in $\operatorname{Hom}(\operatorname{Ihom}(X, I)$, $\operatorname{lnom}(X, I))$. It is obvious that $\left(\operatorname{Ihom}(X, I), \mathrm{ev}_{X}\right)$ is universal with this property. By definition, a left dual to an object $X$ is a pair $\left(X^{*}, \mathrm{ev}_{X}: X^{*} \otimes X \longrightarrow I\right)$ such that there exists a morphism $\mathrm{db}_{X}: I \longrightarrow X \otimes X^{*}$ making the following diagrams commutative:
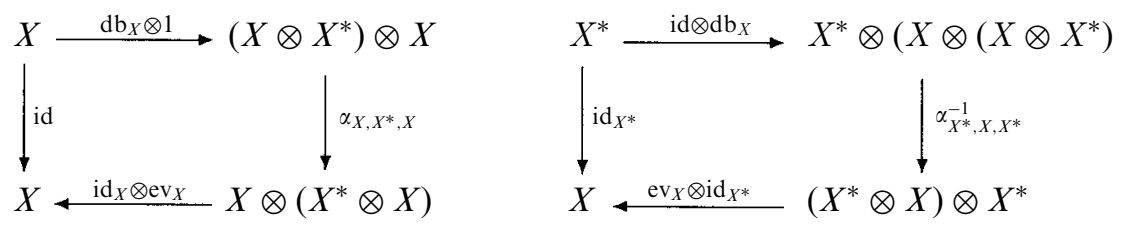

The left dual, if it exists, is uniquely determined up to an isomorphism. In this case, we can identify it with $\operatorname{lhom}(X, I)$. Moreover, we have a natural isomorphism

$$
\operatorname{lnom}(X, Z) \cong Z \otimes X^{*} .
$$

In particular, the functor $\operatorname{Ihom}(X,-)$ exists if $X$ has a left dual. The diagrams in (9) also imply that the functor $X \otimes-$ has a left adjoint: $X^{*} \otimes-$, consequently $X \otimes-$ commutes with colimits.

The definition of a right dual ${ }^{*} X$ to $X$ is similar. Analogous assertions hold for objects having right dual. If ${ }^{*} X$ exists, we can identify it with $\operatorname{rhom}(X, I)$ and we have $\operatorname{rhom}(X, Z) \cong{ }^{*} X \otimes Z$. An object in $\mathrm{A}$ is called rigid if it possesses left and right duals. The category $A$ is called rigid if its objects are rigid. 


\subsection{MONOIDAL FUNCTORS}

Let $(\mathrm{A}, \otimes, I, \alpha, \rho, \lambda)$ and $(\overline{\mathrm{A}}, \bar{\otimes}, \bar{I}, \bar{\alpha}, \bar{\rho}, \bar{\lambda})$ be monoidal categories. A monoidal functor $\mathrm{A} \longrightarrow \overline{\mathrm{A}}$ consists of a functor $\mathrm{F}: \mathrm{A} \longrightarrow \overline{\mathrm{A}}$, a natural ismorphism $\xi_{X, Y}: \mathrm{F}(X) \bar{\otimes}(Y) \longrightarrow$ $\mathrm{F}(X \otimes Y)$ and an isomorphism $\eta: \bar{I} \longrightarrow \mathrm{F}(I)$, satisfying the following commutative diagrams:

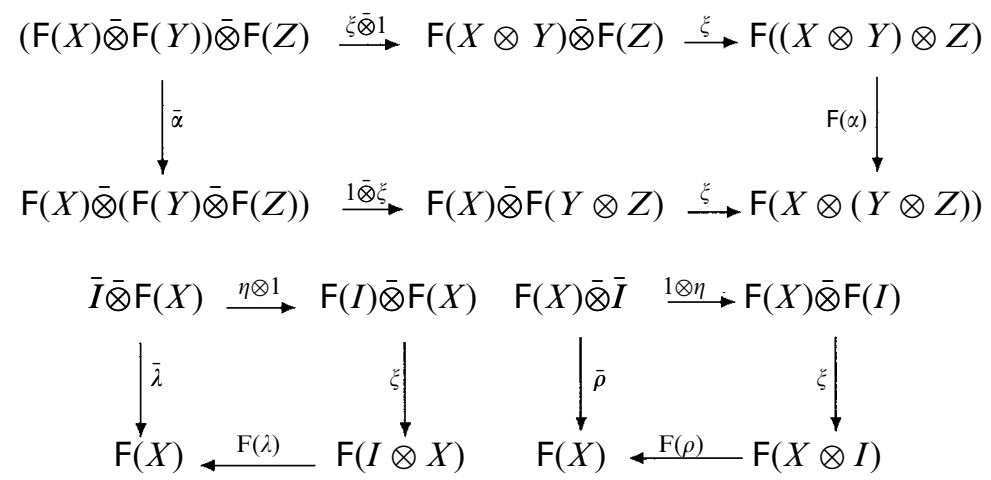

If an object $X$ in $\mathrm{A}$ is rigid, then its image $\mathrm{F}(X)$ is rigid in $\overline{\mathrm{A}}$.

\subsection{ABELIAN MONOIDAL CATEGORIES}

A monoidal category $(A, \otimes)$ is called Abelian monoidal if it is Abelian and the tensor product is an additive bifunctor. In this case, $K:=\operatorname{End}(I)$ is a commutative ring and $\operatorname{Hom}(X, Y)$ becomes $K-K$-bimodule, for any objects $X, Y$. Notice that the two actions of $K$ do not generally coincide and A is therefore not necessarily $K$-linear.

From the discussion in 1.2, if the functor $\operatorname{lnom}(X,-)$ is defined, then it is left exact and the functor $X \otimes-$ is right exact. The same holds for $\operatorname{rhom}(X,-)$ and $-\otimes X$. If $\mathrm{A}$ is closed, then the contravariant functors $\operatorname{Ihom}(-, Z)$ and $\operatorname{rhom}(-, Z)$ are also left exact. If an object $X$ has left dual, then the functor $X \otimes-$ is left exact; hence exact. In particular, if $X$ is rigid, then all the mentioned above functors are exact. Since $X^{*}=\operatorname{lnom}(X, I)$, for a short exact sequence of rigid objects $0 \longrightarrow X \longrightarrow$ $Y \longrightarrow Z \longrightarrow 0$ its dual sequence $0 \longrightarrow Z^{*} \longrightarrow Y^{*} \longrightarrow X^{*} \longrightarrow 0$ is also exact.

\subsection{EXAMPLE: THE CATEGORY OF BIMODULES OVER A RING}

The category of bimodules over a ring is one of the most important examples of monoidal categories. It turns out that this is a very general model of Abelian monoidal category. In fact, as we will see in the next section, any closed Abelian monoidal category can be exactly embedded in a bimodule category. In this subsection, we show some formulae for the functors Ihom and rhom. 
Fix a ring $R$ (or more general a $k$-algebra $R, k$ is a commutative ring). Consider the category of $R-R$-bimodules (for the case of $k$-algebra, we assume that everythings are $k$-linear). It is well known that the tensor product over $R$ makes ${ }_{R} \operatorname{Mod}_{R}$ into an Abelian monoidal category. In particular, the associative constraint is given by the identification $(a \otimes b) \otimes c \longmapsto a \otimes(b \otimes c)$. The unit object is $R$ with the natural actions. We have the following relations between the tensor product and the Hom-functor:

$$
\begin{aligned}
{ }_{R} \operatorname{Hom}_{R}\left(M \otimes_{R} N, P\right) & \cong{ }_{R} \operatorname{Hom}_{R}\left(M, \operatorname{Hom}_{R}(N, P)\right) \\
f & \longmapsto \bar{f}:(\bar{f}(m))(n)=f(m \otimes n), \\
& \cong{ }_{R} \operatorname{Hom}_{R}\left(N,{ }_{R} \operatorname{Hom}(M, P)\right) \\
f & \longmapsto \tilde{f}:(\tilde{f}(n))(m)=f(m \otimes n) .
\end{aligned}
$$

These isomorphisms are natural in all arguments. Thus, we immediately conclude that

$$
\begin{aligned}
& \operatorname{lnom}(N, P) \cong \operatorname{Hom}_{R}(N, P), \\
& \operatorname{rhom}(N, P)={ }_{R} \operatorname{Hom}(N, P) .
\end{aligned}
$$

The following lemma characterizes rigid bimodules and will be used in Section 4.

LEMMA 1.3. Let $M$ be a rigid bimodule. Then $M$ considered as left or right $R$-module is projective of finite type.

Proof. According to (10), we have

$$
\operatorname{Hom}_{R}(M, M) \cong M \otimes \operatorname{Hom}_{R}(M, R) .
$$

Assume that under this isomorphism, id ${ }_{M}$ is mapped to $\sum_{i=1}^{n} m_{i} \otimes \varphi^{i}$, where $\varphi^{i} \in \operatorname{Hom}_{R}(M, R)$, we have the following identity, which follows from the first diagram in (9): $\sum_{i=1}^{n} m_{i} \varphi^{i}(m)=m$, for all $m \in M$. Define the following right $R$-module homomorphisms

$$
\begin{aligned}
& M \longrightarrow R^{n}, \quad m \longmapsto\left(\varphi^{1}(m), \varphi^{2}(m), \ldots, \varphi^{n}(m)\right), \\
& R^{n} \longrightarrow M, \quad\left(r_{1}, r_{2}, \ldots, r_{n}\right) \longmapsto \sum_{i=1}^{n} m_{i} r_{i} .
\end{aligned}
$$

It follows from the previous identity that $M$ is a direct summand of $R^{n}$, that is, projective of finite type. Analogously, using isomorphism

$$
{ }_{R} \operatorname{Hom}(M, M) \cong{ }_{R} \operatorname{Hom}(M, R) \otimes M
$$

and the second diagram in (9) we can show that $M$ is a left projective $R$-module of finite type. 


\section{Tensor Structures on Module Categories}

Let $R$ be a ring and $\operatorname{Mod}_{R}$ be the category of right modules over $R$. Let $\odot$ be a tensor product on $\operatorname{Mod}_{R}$ with associativity constraint $\alpha$ and left-, right units $\lambda, \rho$. We assume that the tensor product is (left and right) closed. That is, (cf 1.1) the functors $-\odot X$ and $X \odot-$ possess right adjoints. Consequently, these functors preserves colimits (cf. Lemma 1.1).

Consider the functor $-\odot X: \operatorname{Mod}_{R} \longrightarrow \operatorname{Mod}_{R}$, which preserves the colimits. By a theorem of Watts [15], there exists a left action of $R$ on $R \odot X$ making it an $R-R$-bimodule, such that the functor $-\odot X$ is naturally equivalent to the functor $-\otimes(R \odot X): Y \longmapsto Y \otimes(R \odot X)$, where $\otimes$ denotes the tensor product over $R$. That is, there is a natural isomorphism

$$
\theta_{X}(Y): Y \otimes(R \odot X) \longrightarrow Y \odot X
$$

For the case $X=R$, we shall call the action of $R$ on $T:=R \odot R$ the second left action of $R$ on $T$, to distinguish it from the first left action subsequently defined.

Explicitly, the left action of $R$ on $R \odot X$ is given as follows:

$$
\begin{aligned}
-\odot \operatorname{id}_{X}: \operatorname{End}_{R}(R) \cong R & \longrightarrow \operatorname{End}_{R}((R \odot X) .) \\
f & \longrightarrow f \odot \operatorname{id}_{X} .
\end{aligned}
$$

Since $\odot$ is biadditive, we see that $\theta_{X}(Y)$ is also natural on $X$. Hence $R \odot-$ : $X \longmapsto R \odot X$ is a functor form the category $\operatorname{Mod}_{R}$ to ${ }_{R} \operatorname{Mod}_{R}$, commuting with colimits. Applying Watts' theorem again, we have an equivalence of the functors $-\odot R$ and $-\otimes T$ with a left action of $R$ on $T$, called the first left action:

$$
\mu_{X}: X \otimes_{1} T \longrightarrow R \odot X
$$

where the subindex 1 indicates that the first left action of $R$ on $T$ is used to define the tensor product. The first left action of $R$ on $T$ is explicitly given as follows:

$$
\begin{aligned}
\operatorname{id}_{R} \odot-: \operatorname{End}_{R}(R) \cong & R \longrightarrow{ }_{R} \operatorname{End}_{R}(R \odot R)={ }_{R} \operatorname{End}_{R}(T) \\
& f \longmapsto \operatorname{id}_{R} \odot f .
\end{aligned}
$$

By its definition, the first left action commutes with the other actions of $R$ on $T$, making $T$ an object in ${ }_{\left(R \otimes_{Z} R\right)} \operatorname{Mod}_{R}$. We have an $R$-linear natural isomorphism

$$
c_{X, Y}:=\theta_{Y}(X) \circ\left(\operatorname{id}_{X} \otimes \mu_{Y}\right): X \otimes_{2}\left(Y \otimes_{1} T\right) \longrightarrow X \odot Y .
$$

The associativity constraint $\alpha$ induces an $R$-linear natural isomorphism

$$
\begin{aligned}
\alpha_{X, Y, Z}^{\prime}:= & \left(\mathrm{id} \otimes c_{Y, Z}\right) c_{X, Y \odot Z}^{-1} \alpha_{X, Y, Z} c_{X \odot Y, Z}\left(c_{X, Y} \otimes \mathrm{id}\right): \\
& \left(X \otimes_{2}\left(Y \otimes_{1} T\right)\right) \otimes_{2}\left(Z \otimes_{1} T .\right) \longrightarrow X \otimes_{2}\left(\left(Y \otimes_{2}\left(Z \otimes_{1} T\right)\right) \otimes_{1} T .\right),
\end{aligned}
$$

where the action of $R$ is induced from the right action on $T$ (indicated by a dot). 
Analogously, we have $R$-linear natural isomorphisms $\lambda^{\prime}$ and $\rho^{\prime}$

$$
\begin{aligned}
& \lambda_{X}^{\prime}:=\lambda_{X} \circ c_{I, X}: I \otimes_{2}\left(X \otimes_{1} T\right) \longrightarrow X, \\
& \rho_{X}^{\prime}:=\rho_{X} \circ c_{X, I}: X \otimes_{2}\left(I \otimes_{1} T\right) \longrightarrow X .
\end{aligned}
$$

Thus, we have defined data for a monoidal structure on $\operatorname{Mod}_{R}$, namely, the tensor product of two module $X, Y$ is $X \otimes_{2}\left(Y \otimes_{1} T\right)$ ), the associative constraint is $\bar{\alpha}$ and the left and right units is $\lambda^{\prime}$ and $\rho^{\prime}$. Using the naturality of $c$, we can show that these data define a monoidal structure on $\operatorname{Mod}_{R}$. More explicitly, using routine diagram chasing, we can show the following lemma.

LEMMA 2.1. Let $(\mathrm{A}, \otimes, I, \alpha, \rho, \lambda)$ be a monoidal category. Let $\odot$ be another bifunctor $\mathrm{A} \times \mathrm{A} \longrightarrow \mathrm{A}$, which is equivalent to $\otimes$ by means of a natural isomorphism $\mathrm{c}$. Then $\odot$ together with the isomorphism $\bar{\alpha}, \rho^{\prime}$ and $\lambda^{\prime}$ defined as in (21), (22) and (23), define another monoidal structure on $\mathrm{A}$.

Setting $X=Y=Z=R$ in (21), we obtain an isomorphism of $R \otimes_{\mathbb{Z}} R \otimes_{\mathbb{Z}} R-$ $R$-bimodules

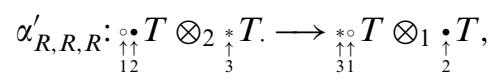

where $\bullet, \circ, *$ denote the different actions of $R$ on the source and the target of $\Psi$. They will be referred to as the first, second, and third left actions of $R$. The right action is indicated by. The following lemma shows that $\alpha^{\prime}$ can be restored from this isomorphism.

LEMMA 2.2. Let $P, Q$ be $R-S$-bimodules. Then a natural transformation $c:-\otimes P \longrightarrow-\otimes Q$ of functors $\operatorname{Mod}_{R} \longrightarrow \operatorname{Mod}_{S}$ is given by an $R-S$-bimodule homomorphism $c=c_{R}: P \longrightarrow Q$.

Proof. We have the following commutative diagram:

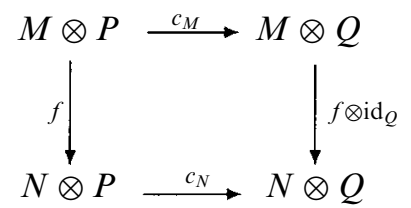

For $N=M=R$ and a morphism $f_{s}: R \longrightarrow R$ or right $R$-modules, $f_{s}(r):=s r$, we have

$$
c(s p)=c_{R}(s \otimes p)=s\left(c_{R}(1 \otimes p)\right)=s c(p),
$$

i.e. $c$ is a left $R$-module morphism. By definition, $c$ is a right $S$-module morphism, hence it is an $R-S$-bimodule morphism. Now let $M=R$ and $N$ be arbitrary. For $n \in N$, choose $f_{n}: R \longrightarrow N, f_{n}(s)=n s$, thus $f$ is a morphism of right $R$-modules. Then 
we have, plugging $f$ and $c_{N}$ in the above diagram, $c_{N}(n \otimes p)=\left(f \otimes \mathrm{id}_{Q}\right) c_{R}(1 \otimes p)=$ $n \otimes c(p)$.

Setting $X=R$ and $Y=I$ in (23), we have a natural isomorphism

$$
\rho^{\prime}:=\rho \circ c_{R, I}: I \otimes_{1} T \longrightarrow R .
$$

Analogously, we have a natural isomorphism

$$
\lambda^{\prime}:=\lambda \circ c_{I, R}: I \otimes_{2} T \longrightarrow R .
$$

The coherent constraints (1), (2), imply the following condition for the isomorphisms $\alpha^{\prime}, \lambda^{\prime}, \rho^{\prime}$ :

$$
\begin{aligned}
& \left(\alpha^{\prime} \otimes_{1} \mathrm{id}_{T}\right)\left(\mathrm{id}_{T} \otimes_{1} \alpha^{\prime}\right)\left(\alpha^{\prime} \otimes_{2} \mathrm{id}_{T}\right)=\left(\mathrm{id}_{T} \otimes_{3} \alpha^{\prime}\right)\left(\mathrm{id}_{T} \otimes_{2} \alpha^{\prime}\right), \\
& \left(\lambda^{\prime} \otimes_{1} \mathrm{id}_{T}\right)\left(\rho^{\prime} \otimes_{2} \mathrm{id}_{T}\right)=\mathrm{id}_{I} \otimes_{1} \alpha^{\prime} .
\end{aligned}
$$

For any right $R$-modules $M, N$, we have a sequence

$$
\begin{aligned}
& \operatorname{Hom}_{R}(M, N) \stackrel{\mathrm{id}_{M} \otimes-}{\longrightarrow} 2 \operatorname{Hom}_{R-R}\left(M \otimes_{1} T ., N \otimes_{1} T .\right) \stackrel{\mathrm{id}_{I} \otimes-}{\longrightarrow} \operatorname{Hom}_{R}(M, N .) \\
& f \longmapsto f \otimes \mathrm{id}_{T} \longmapsto \mathrm{id}_{I} \otimes f \otimes \mathrm{id}_{T}=f .
\end{aligned}
$$

Here ${ }_{2} \mathrm{Hom}_{R}\left(M \otimes_{1} T ., N \otimes_{1} T\right.$.) denotes the set of $R-R$-bimodule morphisms, the left actions of $R$ on whose source and target are given by the second left action of $R$ on $T$. According to the isomorphism in (22), the composition of the above morphisms is an isomorphism, hence the map

$$
\operatorname{Hom}_{R}(M, N) \longrightarrow{ }_{2} \operatorname{Hom}_{R}\left(M \otimes_{1} T, N \otimes_{1} T\right)
$$

is injective.

Consider now the functor $\omega: \operatorname{Mod}_{R} \longrightarrow{ }_{R} \operatorname{Mod}_{R}, X \longmapsto X \otimes_{1} T$, where the left action of $R$ on $X \otimes_{1} T$ is given by the second left action of $R$ on $T$. We have a natural isomorphism

$$
\begin{aligned}
& \xi_{X, Y}:=\alpha_{R, X, Y}^{\prime}:\left(X \otimes_{1} \bullet T\right) \otimes_{2}\left(Y \otimes_{1} T .\right) \longrightarrow\left(X \otimes_{2}\left(\left(Y \otimes_{1} T\right)\right) \otimes_{1} \bullet T .\right. \\
& \omega(X) \otimes \omega(Y) \quad \omega\left(X \otimes_{2}\left(Y \otimes_{1} T\right)\right), \\
& \eta=\rho^{-1}: \bullet R . \longrightarrow I \otimes_{1} \bullet T .
\end{aligned}
$$


LEMMA 2.3. With the notation as above, $(\omega, \xi)$ is a monoidal functor from the monoidal category $\left(\operatorname{Mod}_{R},-\otimes_{2}\left(-\otimes_{1} T\right), I, \alpha^{\prime}, \lambda^{\prime}, \rho^{\prime}\right)$ to the bimodule category $\left({ }_{R} \operatorname{Mod}_{R}, \otimes\right)$.

Proof. One has to check the diagrams in (11) for $\xi$ and $\eta$ given in (31). Plugging $\xi_{X, Y, Z}=\alpha_{R, X, Y, Z}^{\prime}$ into the hexagon in (11) and noticing that in ${ }_{R} \operatorname{Mod}_{R}$, the associativity constraint can be considered as the identity map, we get a pentagon for $\alpha^{\prime}$ (the morphism $\bar{\alpha}$ of the hexagon in (11) reduces to the identity map). In turns out that (by virtue of Lemma 2.2), the commutativity of this pentagon follows from (27) or, in other words, this pentagon for $\alpha^{\prime}$ is precisely the pentagon in (1) for $\alpha^{\prime}$ and $R, X, Y, Z$. Analogously, the first square in (11) for $\eta$ follows from (28) or the triangle in (2) for $\alpha^{\prime}$. Finally, the second square in (11) for $\eta$ follows from the second triangle in (3).

Remark. Although our monoidal category $\left(\operatorname{Mod}_{R}, \odot\right)$ is not assumed to be strict, the functor $\omega$ maps it into an almost strict monoidal category.

Solving Equations (27) and (28) allows us to classify closed monoidal structures on $\operatorname{Mod}_{R}$. This seems to be an interesting problem.

THEOREM 2.4. Let $\odot$ be a monoidal structure on $\operatorname{Mod}_{R}$. Then the functor $\omega: \operatorname{Mod}_{R} \longrightarrow{ }_{R} \operatorname{Mod}_{R}, X \longmapsto X \otimes_{1} T$ is a monoidal right exact embedding.

Proof. We have seen that $\omega$ is monoidal, faithful, and right exact. It remains to show that $\omega(X) \nRightarrow 0$ whenever $X \not 0$. That is, $X \odot R \neq 0$ for all $X \neq 0$. Let $X$ be such that $X \odot R \cong 0$. Then

$$
0=\operatorname{Hom}(X \odot R, X) \cong \operatorname{Hom}(R, \operatorname{rhom}(X, X)) .
$$

Therefore, $\operatorname{rhom}(X, X)=0$. But then

$$
\operatorname{Hom}(X, X) \cong \operatorname{Hom}(I, \operatorname{rnom}(X, X))=0 .
$$

Thus, $X \cong 0$.

Let now $\mathrm{A}$ be a monoidal category which, as an Abelian category, is cocomplete with a progenerator (i.e. small projective generator [11]) and, as a monoidal category, is closed. Let $P$ be a progenerator of A. Then $A$ is equivalent to $\operatorname{Mod}_{R}$, where $R=\operatorname{End}(P)$, by the functor $\mathrm{F}=\operatorname{Hom}(P,-)$. Since $\mathrm{F}$ is an equivalence, it carries the monoidal structure on $A$ over to $\operatorname{Mod}_{R}$. Thus, we have

COROLLARY 2.5. Let $(\mathrm{A}, \odot)$ be an Abelian monoidal category, which is cocomplete and closed and has a progenerator $P$. Then the functor $\omega: X \longmapsto \operatorname{Hom}(P, P \odot X)$ is a monoidal functor from $\mathrm{A}$ to ${ }_{R} \operatorname{Mod}_{R}$, where $R=\operatorname{End}(P)$. This functor is a right exact embedding. It is exact if $P$ is left flat with respect to the tensor product on $\mathrm{A}$. An analogous assertion holds for the functor $X \longmapsto \operatorname{Hom}(P, X \odot P)$. 
Remark. For an object $X$ in A, the actions of $R$ on $\omega(X)$ are given as follows. For $\lambda \in R=\operatorname{End}(P), f \in \omega(X)=\operatorname{Hom}(P, P \odot X)$,

$$
\lambda f=\left(\lambda \odot \mathrm{id}_{X}\right) f, \quad f \lambda=f \circ \lambda .
$$

Since $P$ is a generator, i.e., $\operatorname{Hom}(P, X) \neq 0$ for all $X \not 0$, the functor $\omega$ is exact if and only if $R$ is flat with respect to the tensor product $\odot$. This is not alway the case. Take, for example, the category ${ }_{S} \operatorname{Mod}_{S}$, where $S$ is a ring not flat over $\mathbb{Z}$. Then for $R=S^{\mathrm{op}} \otimes_{\mathbb{Z}} S,{ }_{S} \operatorname{Mod}_{S}$ is equivalent to $\operatorname{Mod}_{R}$. The tensor product is taken over $S$, therefore $M \otimes_{S} R=M \otimes_{S}\left(S^{\mathrm{op}} \otimes_{\mathbb{Z}} S\right) \cong M \otimes_{\mathbb{Z}} S$. Thus $R$ is not flat.

\section{An Embedding Theorem for Small Abelian Monodial Categories}

Using the result of the previous section, we show in this section that a small Abelian monoidal category with exact tensor product can be embedded in the category of bimodules over a ring. Our tactic is to embed $\mathrm{C}$ in a bigger category which is cocomplete with a projective generator.

In the first three paragraphs of this section, we recall the notion of ind-categories. This will be the main ingredient for our embedding. References on ind-categories are [1] and [2, Appendix].

\subsection{THE CATEGORY Ind - C}

A category I is called a filtering category if to every pair $i, i^{\prime}$ of objects from I there exists an object $i^{\prime \prime}$, such that $\operatorname{Hom}\left(i, i^{\prime \prime}\right)$ and $\operatorname{Hom}\left(i^{\prime}, i^{\prime \prime}\right)$ are both not empty, and for every pair of morphisms $f, f^{\prime}: i \longrightarrow i^{\prime}$, there exists a morphism $g: i^{\prime} \longrightarrow j$ equalizing them, i.e. $g f=g f^{\prime}$.

Let $\mathrm{C}$ be an Abelian category. The category Ind $-\mathrm{C}$ of ind-objects of $\mathrm{C}$ consists of functors $\mathrm{X}: \mathrm{I} \longrightarrow \mathrm{C}$, where $\mathrm{I}$ is any small filtering category. Alternatively, denoting $X_{i}:=\mathrm{X}(i), i \in \mathrm{I}$, an ind-object of $\mathrm{C}$ is a directed system indexed by a small filtering category I. For two objects $X=\left\{X_{i}\right\}_{i \in \mathrm{I}}$ and $\mathrm{Y}=\left\{Y_{j}\right\}_{j \in \mathrm{J}}$, their hom-set is

$$
\operatorname{Hom}(\mathrm{X}, \mathrm{Y}):=\lim _{\overleftarrow{i}}\left(\lim _{\vec{j}} \operatorname{Hom}\left(X_{i}, Y_{j}\right)\right) \text {. }
$$

The following lemma will be useful when dealing with hom-sets of ind-objects:

LEMMA 3.1 (cf. [2, Appendix, Cor. 3.2]). Let $\mathrm{X}, \mathrm{Y}$ be ind-objects and $f: \mathrm{X} \longrightarrow \mathrm{Y}$ a morphism. Then there is a cofinial functor $\kappa: \mathrm{I} \longrightarrow \mathrm{J}$, such that $f$ can be represented, up to isomorphism, by a small filtering system of morphisms $\left\{f_{i}: X_{i} \longrightarrow Y_{\kappa(i)}\right\}_{i \in 1}$.

A functor $\kappa$ between filtering systems $\mathrm{I}, \mathrm{J}$ is called cofinal if, for any $j \in \mathrm{J}$, there is an $i \in \mathrm{I}$ and a morphism $j \longrightarrow \kappa(i)$ and for $j \in \mathrm{J}, i \in \mathrm{I}$ and two morphism 
$j \longrightarrow \kappa(i)$, there is a morphism $i \longrightarrow i^{\prime}$ such that the composed morphisms $j \longrightarrow \kappa\left(i^{\prime}\right)$ are equal.

$\mathrm{C}$ is fully embedded in Ind $-\mathrm{C}$ by a constant functor. On the other hand, Ind - C is fully embedded in Fun( $C^{o p}$, Set). For an ind-object $X=\left\{X_{i}\right\}_{i \in \mathrm{I}}$, define the functor

$$
\mathrm{Lx}_{\mathrm{x}} Y \longrightarrow \lim \operatorname{Hom}\left(Y, X_{i}\right) .
$$

By Yoneda's Lemma and the fact that $\operatorname{Hom}(-, X)$ commutes with colimits, we have $\operatorname{Hom}(X, Y) \cong \operatorname{Hom}\left(L_{X}, L_{Y}\right)$. In fact, from definition, $L_{X}$ is isomorphic to $\lim _{\rightarrow} L_{X_{i}}$ in Fun $\left(C^{o p}\right.$, Set). Therefore,

$$
\begin{aligned}
& \operatorname{Hom}\left(L_{X}, L_{Y}\right)=\lim _{i} \operatorname{Hom}\left(L_{X_{i}}, L_{Y}\right), \\
& \text { (by Yoneda's Lemma) }=\underset{\leftarrow}{\lim } L_{Y}\left(X_{i}\right) \\
& =\underset{i}{\lim }\left(\underset{\vec{j}}{\lim } \operatorname{Hom}\left(X_{i}, Y_{j}\right)\right) .
\end{aligned}
$$

The category Ind - C is closed under filtering direct limits (cf. [2, Appendix. 4.4] or $[1, \mathrm{I} .8])$.

Note, however, that the direct limit computed in C (if it exists) is generally different from the one computed in Ind $-\mathrm{C}$.

\subsection{EXTENSION OF FUNCTORS}

Let $F: \mathrm{C} \longrightarrow \mathrm{D}$ be a functor. A functor Ind $-\mathrm{F}:$ Ind $-\mathrm{C} \longrightarrow$ ind $-\mathrm{D}$ is defined as follows:

$$
\text { Ind }-\mathrm{F}\left(\left\{X_{i}\right\}_{i \in \mathrm{I}}\right):=\left\{F\left(X_{i}\right)\right\}_{i \in I}
$$

The action of Ind - F on hom-sets is defined in a straightforward manner:

$$
\begin{aligned}
& \text { Ind }-\mathrm{F}: \operatorname{Hom}(\mathrm{X}, \mathrm{Y})=\underset{\leftarrow}{\lim }\left(\underset{\vec{j}}{\lim } \operatorname{Hom}\left(X_{i}, Y_{j}\right)\right) \longrightarrow \\
& \operatorname{Hom}(\operatorname{Ind}-\mathrm{F}(\mathrm{X}), \text { Ind }-\mathrm{F}(\mathrm{Y}))=\underset{\leftarrow}{\lim }\left(\lim _{\vec{j}} \operatorname{Hom}\left(f\left(X_{i}\right), f\left(Y_{j}\right)\right)\right) .
\end{aligned}
$$

According to [1, Pro I.8.6.3], Ind - F commutes with directed inductive limits.

\subsection{IND-CATEGORY FOR ABELIAN CATEGORIES}

Assume now that $\mathrm{C}$ is Abelian. Then the functor $\mathrm{L}_{X}$ is left exact for any ind-object $\mathrm{X}=\left\{X_{i}\right\}_{i \in \mathrm{I}}$. Indeed, in the category of sets, the filtering direct limits preserves left exact sequences, hence for a left exact sequence in $\mathrm{C}^{\mathrm{op}}: 0 \longrightarrow Y \longrightarrow Y^{\prime} \longrightarrow Y^{\prime}$, we have the following left exact sequences:

$$
0 \longrightarrow \operatorname{Hom}\left(Y, X_{i}\right) \longrightarrow \operatorname{Hom}\left(Y^{\prime}, X_{i}\right) \longrightarrow \operatorname{Hom}\left(Y^{\prime \prime}, X_{i}\right),
$$


$i \in \mathrm{I}$. Taking the limit, we have

$$
0 \longrightarrow \mathrm{L}_{x}(Y) \longrightarrow \mathrm{L}_{x}\left(Y^{\prime}\right) \longrightarrow \mathrm{L}_{x}\left(Y^{\prime}\right) .
$$

Conversely, let $\mathrm{L}$ be a functor $\mathrm{C}^{\mathrm{op}} \longrightarrow \mathrm{Ab}$. By Yoneda's Lemma, for any $X \in \mathrm{C}$, $\operatorname{Hom}(\operatorname{Hom}(-, X), \mathrm{L}(-)) \cong \mathrm{L}(X)$. Consider the system $C_{\mathrm{L}}:=\{(X, \eta) \mid X \in \mathrm{C}$, $\eta \in \mathrm{L}(X)\}$. Morphisms $(X, \eta) \longrightarrow\left(X^{\prime}, \eta^{\prime}\right)$ are those from $\operatorname{Hom}\left(X, X^{\prime}\right)$, which commute with $\eta, \eta^{\prime}$, in the sense that $\mathrm{L}(f)\left(\eta^{\prime}\right)=\eta$. Then $\mathrm{L}$ is isomorphic to the functor

$$
Y \longmapsto \lim _{(X, \eta) \in C_{L}} \operatorname{Hom}(Y, X)
$$

If $\mathrm{L}$ is left exact, then $C_{\mathrm{L}}$ is a filtering system. In fact, given $(X, \eta)$ and $\left(X^{\prime}, \eta^{\prime}\right)$, since left exact functors preserve direct sums, we can take the object $\left(X^{\prime \prime}, \eta^{\prime \prime}\right)$ to be $\left(X \oplus X^{\prime}, \eta \oplus \eta^{\prime}\right)$. For any two morphisms $f, f^{\prime}:(X, \eta) \longrightarrow\left(X^{\prime}, \eta^{\prime}\right)$, let $g: X^{\prime} \longrightarrow Y$ be the coequalizer of $f$ and $f^{\prime}$. Since $\mathrm{F}$ is left exact (from $\mathrm{C}^{\mathrm{op}}$ to $\mathrm{Ab}$ ), we can take $\zeta:=\mathrm{L}(g)^{-1}\left(\eta^{\prime}\right)$. Then $(Y, \zeta)$ is the required pair with $g$ equalizing $f$ and $f^{\prime}$.

Thus, a functor from $C^{o p} \longrightarrow A b$ is left exact if and only if it has the form the same as in (33). The category of left exact functor $\operatorname{Lex}\left(C^{o p}, A b\right)$ is naturally equivalent with Ind-C (cf [2, Appendix 4.5]). We know that the category Lex $\left(\mathrm{C}^{\mathrm{op}}, \mathrm{Ab}\right)$ is a Grothendieck category, i.e. complete, cocomplete with a generator and the filtering limits preserve exact sequences. Injective envelopes exist in such a category and an injective cogenerator exists (cf. [11, Chapter II] or [14, Chapter V, X]).

\subsection{EXTENSION OF MONOIDAL STRUCTURES}

Assume now that $\mathrm{C}$ is a monoidal category. Thus, we have a bifunctor $\odot: \mathrm{C} \times \mathrm{C} \longrightarrow \mathrm{C}$, which induces a bifunctor $\odot$ : Ind $-\mathrm{C} \times$ Ind $-\mathrm{C} \longrightarrow$ Ind $-\mathrm{C}$. Explicitly, for ind-objects $\mathrm{X}=\left\{X_{i}\right\}$ and $\mathrm{Y}=\left\{Y_{j}\right\}$, we set

$$
\mathrm{X} \odot \mathrm{Y}:=\lim _{\vec{i}} \lim _{\vec{j}} X_{i} \odot Y_{j}
$$

It is easy to see that this functor defines a monoidal structure on Ind - C, with the unit object being the unit object in $C$. In fact, for any ind-object $X$, we have:

$$
\mathrm{X} \odot I=\lim _{\vec{i}} X_{i} \odot I=\lim _{\vec{i}} X_{i}=\mathrm{X} .
$$

Assume now that $\mathrm{C}$ is Abelian. Since in Ind $-\mathrm{C}$ directed limits preserve exact sequences, the tensor product in Ind $-\mathrm{C}$ is left (right) exact whenever the tensor product in $C$ is. Indeed, by Lemma 2.2 , any left exact sequence $0 \longrightarrow X \longrightarrow$ $\mathrm{X}^{\prime} \longrightarrow \mathrm{X}^{\prime \prime}$ can be 'uniformly' represented by a filtering system

$$
0 \longrightarrow X_{i} \longrightarrow X_{i}^{\prime} \longrightarrow X_{i}^{\prime \prime}, \quad i \in \mathrm{I} .
$$

Thus, assuming that the tensor product on $\mathrm{C}$ is left exact, for any object $Y_{j}$ of $\mathrm{C}$, the 
sequence

$$
0 \longrightarrow X_{i} \odot Y_{j} \longrightarrow X_{i}^{\prime} \odot Y_{j} \longrightarrow X_{i}^{\prime \prime} \odot Y_{j}, \quad i \in \mathrm{I}, j \in \mathrm{J},
$$

is exact. Since filtering limits preserve exact sequences, we have a left exact sequence

$$
0 \longrightarrow \mathrm{X} \odot Y_{j} \longrightarrow \mathrm{X}^{\prime} \odot Y_{j} \longrightarrow \mathrm{X}^{\prime \prime} \odot Y_{j}, j \in \mathrm{J} .
$$

Taking the limit in $j$, we obtain a left exact sequence

$$
0 \longrightarrow X \odot Y \longrightarrow X^{\prime} \odot Y \longrightarrow X^{\prime \prime} \odot Y .
$$

In particular, the tensor product on Ind $-C$ is exact if the tensor product on $C$ is.

Assume now that $C$ is also rigid. Then objects of $C$ considered as objects of Ind $-\mathrm{C}$ by the constant functor are rigid in Ind $-\mathrm{C}$, too, and the left (right) dual to $X$ is $X^{*}$ (resp. $\left.{ }^{*} X\right)$. Since Ind $-\mathrm{C}$ is complete, i.e., limits exist, the functors Ihom and rhom are definable. In fact, we define

$$
\begin{aligned}
& \operatorname{lnom}(\mathrm{X}, \mathrm{Y}):=\underbrace{\lim \mathrm{Y} \otimes X_{i}^{*}}_{\overleftarrow{i}} \\
& \operatorname{rhom}(\mathrm{X}, \mathrm{Y}):={\underset{\leftarrow}{i}}^{\lim ^{*} X \otimes \mathrm{Y}}
\end{aligned}
$$

The verification is left to the reader. Thus, if $\mathrm{C}$ is rigid, Ind $-\mathrm{C}$ is closed.

\subsection{AN EMBEDDING THEOREM FOR SMALL ABELIAN MONOIDAL CATEGORIES WITH EXACT TENSOR PRODUCT}

Let $C$ be a small, Abelian monoidal category with the exact tensor product. Set $A=$ Ind $-C$. Then we see in the previous section that $A$ is a monoidal Grothendieck category with the exact tensor product. Let $J$ be an injective cogenerator in $A$, which exists due to the fact that $A$ is a Grothendieck category.

Let $R:=\operatorname{End}(J)$. Consider the functor

$$
\operatorname{Hom}(-, J): \mathrm{A}^{\mathrm{op}} \longrightarrow{ }_{R} \operatorname{Mod}
$$

Since $J$ is injective, the functor is exact and since $J$ is a cogenerator, the functor is faithful. Moreover, the functor is full on the full category of subobjects of $J^{\oplus n}$, $n<\infty$ (cf. [11, IV.4.1]). The object $J$ can be chosen so that every object of $\mathrm{C}$ satisfies this condition. Therefore, the induced embedding $\mathrm{C}^{\mathrm{op}} \longrightarrow{ }_{R}$ Mod is exact and fully faithful.

The tensor product on $\mathrm{A}$ induces a bifunctor on a subcategory of ${ }_{R} \mathrm{Mod}$, which contains $R$ - a progenerator of ${ }_{R} \operatorname{Mod}$. Notice that $\operatorname{Hom}_{\mathrm{A}^{\text {op }}}(J, J) \cong{ }_{R} \operatorname{Hom}(R, R)$. Therefore, we can extend the tensor product, which is considered as a functor on the full subcategory of ${ }_{R} \operatorname{Mod}_{R}$, consisting of one object $R$, to a colimit preserving functor on the whole category ${ }_{R} \operatorname{Mod}$ (cf. [11, V.5.2, p106]). The explicit construction is given as follows. 
First, we define the tensor product on the direct sum of $R$. For any sets $S, T$, set $R^{S} \square R^{T}=(R \odot R)^{S \times T}$. Then, for any module $M$, take a resolution

$$
R^{S} \stackrel{f}{\longrightarrow} R^{T} \stackrel{g}{\longrightarrow} M \longrightarrow 0
$$

and define $R^{U} \square M$ to be the cokern of $R^{U} \square f$ :

$$
R^{U} \square R^{S} \longrightarrow R^{U} \square R^{T} \longrightarrow R^{U} \square M \longrightarrow 0 .
$$

Analogously, we define $M \square R^{U}$ and then $M \square N$. Lemma V.5.2.1 of [11] ensures that the above construction does not depend on the choice of resolution. The associativity constraint is defined first on the direct sums of $R$ and then projected onto the other objects.

From the construction of $\square$, we see that if $M$ and $N$ are finitely presented modules, then $M \odot N \cong M \square N$. On the other hand, we know that if an object $X$ of $\mathrm{C}$ has a resolution of the form $0 \longrightarrow X \longrightarrow J^{S} \longrightarrow J^{T}$ where $S, T$ are finite sets, then the $R$-module $M=\operatorname{Hom}(X, J)$ is a finitely presented $R$-module.

This condition may not be satisfied for any injective cogenerator. However, it can be achieved by increasing the cogenerator. We take the direct sum of all objects from $C$ and then take its injective envelope. Denote the object obtained by $J_{1}$. Then $J \oplus J_{1}$ is also an injective cogenerator, in which every object of C can be embedded. For any $X \in \mathrm{C}$, let $i_{X}$ be an embedding in $J \oplus J_{1}$ and let $X^{\prime}$ be the cokern of $i_{X}$, i.e., we have an exact sequence $0 \longrightarrow X \longrightarrow J \oplus J_{1} \longrightarrow X^{\prime} \longrightarrow 0$. Now, let $J_{2}$ be the injective envelope of the direct sum of all $X^{\prime}$ where $X$ runs in C. Let $\bar{J}:=J \oplus J_{1} \oplus J_{2}$. Then, for any $X \in \mathrm{C}$, we have a resolution by $\bar{J}$ :

$$
0 \longrightarrow X \subset \bar{J} \longrightarrow J \oplus X^{\prime} \oplus J_{2} \longleftrightarrow \bar{J} \oplus \bar{J} .
$$

Since $\square$ preserves colimits and since any module is a filtering direct limit of finite presented modules (cf. [14, I.5]), we have, for any $R$-module $M=\lim _{\rightarrow} M_{i}, M_{i}$ are finitely presented $R$-modules,

$$
I \square M \cong I \square \lim _{\vec{i}} M_{i} \cong \lim _{\vec{i}}\left(I \square M_{i}\right) \cong \lim _{\vec{i}} M_{i}=M .
$$

Thus, $I$ is the unit object in ${ }_{R}$ Mod with respect to the tensor product $\square$.

Applying the result of the previous section, we have a monoidal functor

$$
\omega:{ }_{R} \operatorname{Mod} \longrightarrow{ }_{R} \operatorname{Mod}_{R}, \quad M \longmapsto M \square R,
$$

which is a right exact embedding. Compose $\omega$ with the functor $\operatorname{Hom}(-, \bar{J})$, we get a right exact functor from $\mathrm{A}^{\mathrm{op}}$ to ${ }_{R} \operatorname{Mod}_{R}$, whose restriction on $\mathrm{C}^{\mathrm{op}}$ is a right exact monoidal embedding. The last functor is given by $X \longmapsto \operatorname{Hom}(X \odot J, J)$. Thus we have proved:

THEOREM 3.2. Let $\mathrm{C}$ be a small Abelian monoidal category with the tensor product being exact. Then $\mathrm{C}^{\mathrm{op}}$ admits a right exact monoidal embedding into the category 
${ }_{R} \operatorname{Mod}_{R}$ for some ring $R$. The functor is given explicitly by $X \longrightarrow \operatorname{Hom}(J, X \odot J)$ for a suitably chosen injective cogenerator $J$ in Ind - C.

If $\mathrm{C}$ is an Abelian rigid monoidal category then the tensor product is exact and the theorem above applies. In this case we have $\operatorname{Hom}(X \odot J, J) \cong \operatorname{Hom}\left(J,{ }^{*} X \odot J\right)$. Thus, composing the embedding with the contravariant exact functor $X \longrightarrow X^{*}$, we obtain an embedding for $\mathrm{C}: X \longrightarrow \operatorname{Hom}(J, X \odot J)$. Moreover, the embedding is exact. Indeed, let $0 \longrightarrow X \longrightarrow Y \longrightarrow Z \longrightarrow 0$ be an exact sequence. Applying the left dual functor, we have an exact sequence $0 \longrightarrow Z^{*} \longrightarrow Y^{*} \longrightarrow X^{*} \longrightarrow 0$. Let $\mathrm{F}$ denote the embedding functor. Then we have a right exact sequence of $R$-modules:

$$
\mathrm{F}\left(Z^{*}\right) \longrightarrow \mathrm{F}\left(Y^{*}\right) \longrightarrow \mathrm{F}\left(X^{*}\right) \longrightarrow 0 .
$$

Since $\mathrm{F}$ is a monoidal functor, $\mathrm{F}\left(X^{*}\right) \cong \mathrm{F}(X)^{*}=\operatorname{lnom}(\mathrm{F}(X), R)$. Therefore $\mathrm{F}(X) \cong$ $\operatorname{rhom}\left(\mathrm{F}\left(X^{*}\right), R\right)$. Applying the left exact functor $\operatorname{Hom}(-, R)$, we obtain a left exact sequence $0 \longrightarrow \mathrm{F}(X) \longrightarrow \mathrm{F}(Y) \longrightarrow \mathrm{F}(Z)$.

Since the category of bimodules over a ring is cocomplete, the embedding can be extended to a functor Ind $-\mathrm{F}: \operatorname{Ind}-\mathrm{A} \longrightarrow{ }_{R} \operatorname{Mod}_{R}$, which is also exact and monoidal. Explicitly, this functor has the form, for $\mathrm{X}=\left\{X_{i}\right\}_{i \in 1}$ :

$$
\text { Ind }-\mathrm{F}(\mathrm{X})=\lim _{\vec{i}} \operatorname{Hom}\left(J, X_{i} \odot J\right) \text {. }
$$

THEOREM 3.3. Let $\mathrm{C}$ be a small Abelian monoidal rigid category. Then $\mathrm{C}$ admits an exact monoidal embedding into the category of bimodules over a ring. Further, the embedding is extendable to an exact embedding of the category Ind - C, which commutes with colimits.

Proof. What remains to be proved is that the functor Ind $-\mathrm{F}$ is faithful or, equivalently, that ind $-F(X) \neq 0$ whenever $X \neq 0$.

First, we remark that since Ind $-F$ is exact, Ind $-F(X) \nsucceq 0$, whenever $X$ possesses a subobject (or a quotient object) $\mathrm{Y}$, with Ind $-\mathrm{F}(\mathrm{Y}) \neq 0$.

The following fact in Ind $-\mathrm{C}$ is well known (cf. [6, Cor. II.3.2]). If an ind-object $\mathrm{X}$ is a subobject of an object $X \in \mathrm{C}$, then $\mathrm{X}$ contains a subobject $Y \in \mathrm{C}$. Indeed, let $i: \mathrm{X} \longrightarrow X$ be a monomorphism in Ind $-\mathrm{C}$ and $j: Y \longrightarrow \mathrm{X}$ be a nonzero morphism, $Y \in \mathrm{C}$, then $i \circ j: Y \longrightarrow X$ is nonzero and is a morphism in $\mathrm{C}$, for $\mathrm{C}$ is a full subcategory of Ind - C. $j(Y)$ is therefore in $\mathrm{C}$ and, on the other hand, a subobject of $\mathrm{X}$.

A direct consequence of this fact and the preceding remark is that the image under Ind $-\mathrm{F}$ of any nonzero ind-object, which is a subobject of an object from $\mathrm{C}$, is nonzero.

Let now $\mathrm{X} \in \mathrm{Ind}-\mathrm{C}$ be a nonzero object. There exists a nonzero morphism $j: Y \longrightarrow \operatorname{Ihom}(\mathrm{X}, I), Y \in \mathrm{C}$. Since

$$
\operatorname{Hom}\left(\mathrm{X},{ }^{*} Y\right) \cong \operatorname{Hom}(Y \odot \mathrm{X}, I) \cong \operatorname{Hom}(Y, \operatorname{Ihom}(\mathrm{X}, I)),
$$

there exists a nonzero morphism $k: \mathrm{X} \longrightarrow{ }^{*} Y$, corresponding to $j$ in the above 
isomorphisms. Im $k$ is a nonzero subobject of $*$, hence $\operatorname{Ind}-\mathrm{F}(\operatorname{Im} k) \neq 0$, and, consequently, Ind $-\mathrm{F}(\mathrm{X}) \nRightarrow 0$.

\section{Semisimple Abelian Rigid Monoidal Categories}

In this section we consider a simple case, where the construction in the previous section can be explicitly given. Let $\mathrm{C}$ be a small semisimple rigid monoidal category. Thus, objects of $\mathrm{C}$ are direct sums of simple objects $X_{i}, i \in \mathcal{I}$, and the rings $R_{i}=\operatorname{End}\left(X_{i}\right)$ are skew-fields, where $\mathcal{I}$ is a set.

For any object $X \in \mathrm{C}$, we have $\operatorname{End}(X) \cong \operatorname{Hom}\left(I, X \otimes X^{*}\right)$. If $I$ is simple, then the dimension of $\operatorname{End}(X)$ over $K=\operatorname{End}(I)$ is equal to the number of copies of $I$ in the decomposition of $X \otimes X^{*}$.

PROPOSITION 4.1. Let $\mathrm{C}$ be a semisimple rigid monoidal category with a simple unit object. Then, for any object $X \in \mathrm{C}$, $\operatorname{End}(X)$ is finite-dimensional over $\operatorname{End}(I)$, in particular, objects of $\mathrm{C}$ are finite direct sums of simple objects.

Proof. Assume the contrary that $\operatorname{End}(X)$ is infinite dimensional over $K=\operatorname{End}(I)$. Let $e_{s}, s \in S$ be a basis for this space and $f_{s}, s \in S$ be the corresponding morphisms in $\operatorname{Hom}\left(I, X \otimes X^{*}\right)$. Let $F_{s}:=\operatorname{Im}\left(f_{s}\right)$ be subobjects of $X \otimes X^{*}$. The linear independence of $e_{s}, s \in S$ implies that the intersection of any finite subset of $F_{s}$ is zero. Let now $\mathrm{F}$ be an exact monoidal embedding of $\mathrm{C}$ into ${ }_{R} \operatorname{Mod}_{R}$ as in Theorem 3.3. Then the above property of $F_{S}$ is preserved under $F$, that is for any finite subset $K$ of $S \bigcap_{s \in K} \mathrm{~F}\left(F_{S}\right)=0$. This property of submodules $\mathrm{F}\left(F_{S}\right)$ of $\mathrm{F}\left(X \otimes X^{*}\right)$ implies that $\sum_{s \in S} \mathrm{~F}\left(F_{s}\right)=\bigoplus_{s \in S} \mathrm{~F}\left(F_{S}\right)$. On the other hand, since each $F_{S}$ is a direct summand of $X \otimes X^{*} \mathrm{~F}\left(F_{S}\right)$ is a direct summand of $\mathrm{F}\left(X \otimes X^{*}\right)$. Thus $\mathrm{F}\left(X \otimes X^{*}\right)$ contains as direct summand a free module of infinite rank. This contradicts with the fact that $\mathrm{F}\left(X \otimes X^{*}\right)$ is a projective module of finite rank when considered as a left of right $R$-module (cf. Lemma 1.3).

If every object of $C$ is a finite sum of simple object, the category $A=$ Ind $-C$ is easy to characterize. Each object of $\mathrm{A}$ is a direct sum of copies of $X_{i}, i \in \mathcal{I}$. An injective cogenerator can be chosen to be $J=\bigoplus_{i \in \mathcal{I}} X_{i}$. Our embedding $\mathrm{F}$ is then

$$
M \longmapsto \operatorname{Hom}\left(\bigoplus_{j} X_{j}, M \odot\left(\bigoplus_{k} X_{k}\right)\right) \cong \prod_{j} \bigoplus_{k} \operatorname{Hom}\left(X_{j}, M \odot X_{k}\right),
$$

in the category of $R-R$-bimodules, where $R=\operatorname{End}(J) \cong \prod_{i} R_{i}$. Each $R_{i}$ being an endomorphism ring of a simple object is a skew-field (non-commutative field).

Recall that the right action of $R$ on $\mathrm{F}(M)=\operatorname{Hom}(J, M \odot J)$ is given by the composition $(f, \lambda) \longmapsto f \circ \lambda, f \in \mathrm{F}(M), \lambda \in F$, and the left action of $R$ on $\mathrm{F}(M)$ is given by the composition $(\lambda, f) \longmapsto\left(\operatorname{id}_{M} \odot \lambda\right) \circ f$.

By the isomorphism in (38), we see that, as right $R$-module, $\mathrm{F}(M)$ is a direct product of $M^{j}:=\bigoplus_{k} \operatorname{Hom}\left(X_{j}, M \odot X_{k}\right)$, where each $M^{j}$ is a right $R_{i}$-module. On the other 
hand, as left $R$-module, $M^{j}$ is a direct sum of $M^{j}{ }_{k}:=\operatorname{Hom}\left(X_{j}, M \odot X_{k}\right)$, each $M^{j}{ }_{k}$ is a left $R_{k}$-module.

From now on, in addition to the above assumptions on $\mathrm{C}$, we shall assume that $\mathrm{C}$ is $K$-linear, where $K=\operatorname{End}(I)$, and that $\operatorname{End}\left(X_{i}\right) \cong K$ for all simple object $X_{i}$. Thus, for objects $X$ and $Y$, the two actions of $K$ on $\operatorname{Hom}(X, Y)$ coincide, and we shall denote by $h(X, Y)$ the dimension over $K$ of this vector space. Note, however, that the actions of $R=\operatorname{End}(J)$ over on $\operatorname{Hom}(J, M \odot J)$ are generally different.

Let $c_{i k}^{j}$ be the multiplicity of $X_{j}$ in $X_{i} \odot X_{k}$. Then we have

$$
h\left(X_{j}, X_{i} \odot X_{k}\right)=c_{i k}^{j} .
$$

According to Proposition 4.1, for $i, k$ fixed, there are only finitely many $j$ such that $c_{i k}^{j} \neq 0$. On the other hand, since $C$ is semisimple and rigid, we have

$$
h\left(X_{j}, X_{i} \odot X_{k}\right)=h\left(X_{i} \odot X_{k}, X_{j}\right)=h\left(X_{k}, X_{j} \odot X_{i}^{*}\right)=c_{j i^{*}}^{k},
$$

where we use the notation $X_{i^{*}}:=X_{i}^{*}$. Therefore, for $i$ and $j$ fixed, there are only finitely many $k$, such that $c_{i k}^{j} \neq 0$. Analogously, we can show that for $k$ and $j$ fixed, there are only finitely many $i$ such that $c_{i k}^{j}$ is nonzero.

The following equation is easy to check:

$$
h\left(X_{i}, X_{m} \odot X_{n} \odot X_{j}\right)=\sum_{k} h\left(X_{i}, X_{m} \odot X_{k}\right) h\left(X_{k}, X_{n} \odot X_{j}\right) .
$$

In fact, both sides of this equation are equal to $\sum_{k} c_{m k}^{i} c_{n j}^{k}$. Since each object of $\mathrm{C}$ is a direct sum of finitely many simple objects, we deduce

$$
h\left(X_{i}, M \odot N \odot X_{j}\right)=\sum_{k} h\left(X_{i}, M \odot X_{k}\right) h\left(X_{k}, N \odot X_{j}\right)
$$

for all objects $M, N$.

According to Lemma 1.3, $\mathrm{F}(M)$ is projective of finite type as left or right $R$-module. Recall that $R$ is a product of fields $R_{i}$ and as right $R$-module, $\mathrm{F}(M)$ is a product of $R_{i}$-modules.

LEMMA 4.2. Let $R$ be a product of fields $R_{i}, R=\prod_{i} R_{i}$. Let $P=\prod_{i} P_{i}$ be projective of finite rank over $R$. Then the dimension of $P_{i}$ over $R_{i}$ is uniformly bounded by a positive integer.

Proof. For any two $R$-modules of the form $P=\prod_{i} P_{i}, Q=\prod_{i} Q_{i}$, where $P_{i}$ and $Q_{i}$ are $R_{i}$ modules, we have

$$
\operatorname{Hom}_{R}(P, Q)=\prod_{i} \operatorname{Hom}_{R}\left(P, Q_{i}\right)=\prod_{i} \operatorname{Hom}_{R_{i}}\left(P_{i}, Q_{i}\right) .
$$

Let now $P=\prod_{i} P_{i}$ be projective of finite type. Then $P$ is a direct summand of $R^{d}$ for a certain positive integer $d$. In particular, we have a surjective homomorphism $R^{d} \longrightarrow P$. According to (38), we see that for all $i, \operatorname{rank}_{R_{i}} P_{i} \leqslant d$. 
COROLLARY 4.3. Let $R_{i} \cong K$, for all $i$. For any object $M$ in $\mathrm{C}$, the ranks of $M^{j}=\bigoplus_{k} \operatorname{Hom}\left(X_{j}, M \odot X_{k}\right)$ over $K$ are bounded by a certain positive number.

THEOREM 4.4. Let $\mathrm{C}$ be a semisimple Abelian rigid monoidal category with simple unit object, whose endomorphism ring is denoted by $K$. Assume that, for any simple object, its endomorphism ring is isomorphic to $K$. Then for any object $X$, there exists a positive number $d$, such that the dimension over $K$ of $\operatorname{End}\left(X^{n}\right)$ does not exceed $d^{n}$.

Proof. We have

$$
\operatorname{dim}_{K} \operatorname{End}\left(X^{\odot n}\right)=h\left(X^{\odot n}, X^{\odot n}\right)=h\left(I, X^{\odot n} \odot X^{* \odot n}\right) .
$$

Since $I$ is simple, we have

$$
\begin{aligned}
h\left(I, X^{\odot n} \odot X^{* \odot n}\right) & \leqslant \sum_{k} h\left(I, X^{\odot n} \odot X^{* \odot n} \odot X_{k}\right) \\
& \leqslant \max _{i}\left(\sum_{k} h\left(X_{i}, X^{\odot n} \odot X^{* \odot n} \odot X_{k}\right)\right) .
\end{aligned}
$$

For an object $M$ in $\mathrm{C}$, set

$$
d_{M}:=\max _{i}\left(\sum_{k} h\left(X_{i}, M \odot X_{k}\right)\right) .
$$

By virtue of Corollary $4.3, d_{M}$ is well defined, i.e., finite. On the other hand, using (41), we have

$$
\begin{aligned}
\sum_{k} h\left(X_{i}, M \odot N \odot X_{k}\right) & =\sum_{l, k} h\left(X_{i}, M \odot X_{l}\right) h\left(X_{l}, N \odot X k\right) \\
& \leqslant \sum_{l} h\left(X_{i}, M \odot X_{l}\right) d_{N} \leqslant d_{M} d_{N} .
\end{aligned}
$$

Therefore $d_{M \odot N} \leqslant d_{M} d_{N}$. Consequently, $h\left(I, X^{\odot n} \odot X^{* \odot n}\right) \leqslant d_{X}^{n} d_{X^{*}}^{n}=\left(d_{X} d_{X^{*}}\right)^{n}$. The proof is complete.

Theorem 4.4 has the following interesting consequence. First, recall that given a symmetry in $\mathrm{C}$, we can define, for each object $X$, its categorical dimension $\operatorname{dim} X$ to be the morphism $I \stackrel{\mathrm{db}}{\longrightarrow} X \odot X^{*} \stackrel{\tau}{\longrightarrow} X^{*} \odot X \stackrel{\text { ev }}{\longrightarrow} I$, an element of $K=\operatorname{End}(I)$. This dimension is an additive (with respect to direct sums) and tensor-multiplicative function on $X$, cf. [3, Sect. 7].

COROLLARY 4.5. Assume that $\mathrm{C}$ satisfies the condition of Theorem 4.4 and that, moreover, $\mathrm{C}$ is symmetric and its nonzero objects have nonzero dimension. Then, if $\operatorname{char} K=0$, for any object $X$ of $\mathrm{C}$, there exists an integer $n$, for which $\bigwedge_{n}(X)-$ the $n$th antisymmetric tensor power of $X$ is zero. Consequently, C is Tannakian.

Proof. The symmetry induces an algebra homomorphism $\rho_{n}$ from the group algebra $K\left[\Im_{n}\right], \Im_{n}$ is the symmetric group, to $\operatorname{End}\left(X^{\odot n}\right)$ for any object $X$. Since 
the dimension of $K\left[\Im_{n}\right]$ over $K$ is $n$ !, Theorem 4.4 ensures that starting form some $n$, the representation is not faithful. Thus, there exists a minimal central idempotent of $K\left[\subseteq_{n}\right]$ which is mapped to zero by homomorphism $\rho_{n}$.

Since $\operatorname{char} K=0, K\left[\Xi_{n}\right]$ is semisimple and its minimal central idempotents define direct summands of $\operatorname{End}\left(X^{\odot n}\right)$ by means of $\rho_{n}$. It is well known that minimal central idempotents of $K\left[\Im_{n}\right]$ are in one-to-one correspondence with partitions of $n$, see, e.g. [8]. Using the additivity and tensor multiplicativity of the categorical dimension, we can compute the dimension of the direct summand of $X^{\odot n}$ corresponding to a minimal central idempotent in terms of the dimension of $X$ and the corresponding partition. More explicitly, let $X_{\lambda}$ be the direct summand of $X^{\odot n}$ defined by the minimal central idempotent $e_{\lambda}$, where $\lambda$ is a partition of $n$. Then we have

$$
\operatorname{dim} X_{\lambda}=\prod_{\kappa \in[\lambda]} \frac{\operatorname{dim} X+c(\kappa)}{h(\kappa)},
$$

where the product is taken over all blocks $\kappa$ of the diagram [ $\lambda]$ defined by $\lambda$ and $c(\kappa)$ denotes the content of $\kappa$ (the diffencence between the number of boxes left to $\kappa$ and the number of the boxes above $\kappa$ ), $h(\kappa)$ denotes the hook-length of $\kappa$ (the number of boxes right to- and below $\kappa$ including $\kappa$ itself-counted only one time), see also $[10,7]$.

Now, the fact that for some $n$, there exists a partition $\lambda$, such that $e_{\lambda}$ is mapped to zero by $\rho_{n}$, implies that the dimension of $X$ should be an integer. By the assumption that the dimension of any object is nonzero and by the obvious fact that the dimension of the unit object is 1 , it follows that the dimension of any nonzero object is a positive integer. In this case, for an object $M$ with dimension $m$, we have $\operatorname{dim} \bigwedge_{m} X=0$, therefore $\bigwedge_{m} X=0$. The fact that $\mathrm{C}$ is Tannakian now follows from Deligne's theorem [3, Thm. 7.1].

\section{Acknowledgements}

The author would like to thank Professors M. Artin, B. Pareigis and P. Smith for useful discussions. This work is supported by the Mathematical Sciences Research Institute and was presented at the conference on quantum groups in Morelia, Mexico. The author would also like to thank the organizers and the Institute of Mathematics, UNAM for the hospitality during the conference.

\section{References}

1. Artin, M., Grothendieck, A. and Verdier, J. L.: Théorie des topos et cohomologie étale des schémas, In: Séminaire de Géométrie Algébrique du Bois-Marie 1963-1964 (SGA 4), Lecture Notes in Math. 269, Springer, Berlin, 1973.

2. Artin, M. and Mazur, B.: Etale Homotopy, Lecture Notes in Math. 100, Springer, Berlin, 1969.

3. Deligne, P.: Catégories tannakiennes. In: P. Cartier et al. (eds), The Grothendieck Festschrift, volume II, Progr. Math. 87, Birkhäuser, Boston, MA, 1990, pp. 111-195. 
4. Doplicher, S. and Roberts, J. E.: A new duality theory for compact groups, Invent. Math. 98(1) (1989), 157-218.

5. Drinfel'd, V. G.: Quantum groups, Proc. ICM, Berkeley, 1987.

6. Gabriel, P.: Des catégories abéliennes, Bull. Soc. Math. France 90 (1962), 323-448.

7. Phung Ho Hai: Hecke symmetries, J. Pure Appl. Algebra 152 (2000), 109-121.

8. James, G. D.: The Representation Theory of Symmetric Groups, Lecture Notes in Math., Springer, Berlin, 1978.

9. Mac Lane, S.: Categories, for the Working Mathematician, Springer, Berlin, 1971.

10. Macdonald, I. G.: Symmetric Functions and the Hall Polynomials, Oxford Univ. Press, New York, 1979 (2nd edn, 1995).

11. Mitchell, B.: Theory of categories, Pure Appl. Math. 17, Academic Press, New York, 1965.

12. Pareigis, B.: Lectures on quantum groups, Available at www.mathematik.unimuenchen.de/ pareigis, 1994.

13. Schauenburg, P.: The monoidal center construction and bimodules, J. Pure Appl. Algebra 158(2-3) (2001), 325-346.

14. Stenström, B.: Rings of Quotients, Grundlehren Math. Wiss., Springer-Verlag, New York, 1975.

15. Watts, C. E.: Intrinsic characterizations of some additive functors, Proc. Amer. Math. Soc. 11 (1960), 5-8. 